\title{
A time dependent model to study the effect of surface roughness on reactive-diffusive transport in porous media
}

\author{
M. Warrier ${ }^{a}$, A. Rai ${ }^{b}$, R. Schneider ${ }^{b}$ \\ ${ }^{a}$ Computational Analysis Division, Bhabha Atomic Research Center, \\ Trombay, Mumbai, India - 400085, \\ ${ }^{b}$ EURATOM Association, Max-Planck-Institut für Plasmaphysik, Wendelsteinstrasse 1, \\ D-17491 Greifswald, Germany
}

\begin{abstract}
A model for reactive-diffusive transport and recycling of hydrogen from rough graphite surfaces is presented which helps understand the qualitative behavior of hydrogen atomic and molecular release as a function of target temperature and incident hydrogen flux, as observed in experiments and simulations. The effect of surface roughness on the reaction rates of atoms diffusing on the surface is presented. We present reasons for some counterintuitive observations like (i) steady state hydrogen saturation is faster at $600 \mathrm{~K}$ than at $900 \mathrm{~K}$, (ii) the steady state hydrogen density on a rough surface is lesser than that for a smooth surface at a specific target temperature and incident hydrogen flux.
\end{abstract}

JNM keywords: Graphite, Diffusion, Hydrogen, Theory and Modeling.

PSI-17 keywords: Graphite, Plasma Surface Interaction, Hydrogen Recycling PACS codes: 68.55.-a, 68.55.AC, 66.30.-h, 66.30.Jt, 66.30.Ny

${ }^{*}$ Corresponding author address and e-mail: Stellaratortheorie, Max-Planck-Institut für Plasmaphysik, Wendelsteinstrasse 1, D-17491 Greifswald, Germany; Ralf.Schneider@ipp.mpg.de Presenting author name and e-mail: Ralf Schneider: Ralf.Schneider@ipp.mpg.de 


\section{Introduction}

Plasma surface interactions in fusion devices can be classified, based on the underlying physical mechanisms, into momentum transfer processes (physical sputtering, backscattering, etc), diffusive processes (radiation enhanced sublimation) and reactive-diffusive processes (hydrogen and hydrocarbon molecule formation, transport and release). The momentum transfer processes are modeled well using the binary collision approximation (BCA) $[1,2]$. The diffusive and reactive-diffusive processes depend upon the damage, interstitials formed, and, the deposition profiles, all of which result from the momentum transfer processes. These values are usually approximated from a BCA-based Monte Carlo calculation, and are source terms in models for hydrogen recycling from materials [3-6].

We have developed a multi-scale model for reactive-diffusive hydrogen transport in porous materials $[7,8,6,9]$. It is seen that the reactive-diffusive transport on the internal surfaces of porous graphite is an important aspect of hydrogen recycling and retention. Therefore in this paper we report results from a kinetic Monte-Carlo model (KMC) of reactiondiffusion on rough surfaces. This model is described in the next section. The results from this model for smooth and rough surfaces will be presented. Then, a set of particle balance equations based on the ideas underlying $\mathrm{KMC}$ is presented. We compare the results of this analytical model with our simulations. 


\section{KMC model for reactive-diffusive hydrogen transport on rough graphite surfaces}

\subsection{Model}

The model is illustrated in Fig.1. Hydrogen atoms with low energy are incident on a graphite target. These atoms can either

- be solute on the surface and jump from point to point on the surface [10], or

- desorb from the surface and either get out or be incident on a jutting edge if the surface is rough.

The migration energy for the surface jumps is $0.9 \mathrm{eV}$ and the typical jump length is $\sim 34.4$ $\AA$ assuming a jump attempt frequency $\omega_{o}=10^{13} \mathrm{sec}^{-1}[10,11]$. The scale of the roughness curtails the jump length for the surface jumps in our model. The desorption energy of hydrogen from the surface is taken to be $1.9 \mathrm{eV}[12]$ and an atom desorbs with a uniform probability into the $2 \pi$ solid angle accessible to it. On a rough surface it can get adsorbed again on any part of the surface jutting out into its path, whereas on a smooth surface it always gets out of the target.

In any of these two cases, an atom can recombine with another atom to form a hydrogen molecule if the two atoms come closer than a minimum distance for recombination, $R_{o}$. This is called the Smolouchowski boundary condition [13,14]. The hydrogen molecule is very loosely adsorbed on the surface with an adsorption energy $0.06 \mathrm{eV}[3]$. 
Sources (incoming fluxes), sinks (desorbing fluxes), reactions and diffusion limited reactions were implemented in a Kinetic Monte-Carlo ansatz $[15,16]$. For the specific implementation and a description of the underlying equations governing hydrogen reactiondiffusion within the $\mathrm{KMC}$ see $[6,8]$.

We construct the rough surface by first dividing the volume into cells of size $10 \AA$. Then we randomly choose a point and using a Poisson distribution having a mean value of the size of substructure we want to create and populate the cells. We repeat this ansatz till a specified void fraction of the total volume is occupied. Therefore, at most our artificially created structure would not have many self similar features as opposed to a fractal surface. One would have to go to smaller cell-sizes (at most $1 / 5^{\text {th }}$ of the current cell size) and consider truncated levels of self-symmetry to interpret the roughness using fractal dimensions. This is being done and is outside the scope of this paper.

\subsection{Results and discussion}

We carry out simulations at target temperatures of 300, 600, 900 and $1200 \mathrm{~K}$. The sample size facing the incoming hydrogen flux of $10^{24} \mathrm{H}$ atoms $\left(/ \mathrm{m}^{2} / \mathrm{sec}\right)$ was $1000 \times 1000 \AA$ for both, smooth and rough surfaces. The roughness, as characterized by the root mean square deviation along the direction of incoming flux, was $\sim 50 \AA$. The scale-length along the surface of the crests and troughs forming the rough surface was around $\sim 50-100$ $\AA$. The simulations were carried out for 10 million KMC steps. 
In Fig.2 a plot of the approach to steady-state hydrogen density at the different temperatures for a smooth surface is shown. At $300 \mathrm{~K}$, due to the practical limits in the number of particles in the KMC simulation (memory limit), steady state could not be reached. Usually it is expected that at higher target temperature, atomic interactions take place faster and therefore a steady state balance is achieved faster than at lower temperatures. However, we find that a steady state at $600 \mathrm{~K}$ is reached faster than at $900 \mathrm{~K}$ contrary to the naive expectation. This is because desorption, which has an activation energy of $1.9 \mathrm{eV}$ and is a slow process, becomes more and more important at higher temperatures, delaying the approach to the steady state.

Fig. 3 is a plot of the hydrogen retention at $900 \mathrm{~K}$ for a rough and a smooth surface. It is expected that rough surfaces capture the desorbing atoms and therefore the surface density of hydrogen must be higher on a rough surface. It is, however seen, that the density of hydrogen is lower for the rough surface. We observe that there is more $H_{2}$ molecule formation and release in the rough surface case. This will be discussed within an analytic model. The effect of a rough surface on atomic hydrogen recombination can be analyzed using the fractal dimensionality of the rough surface and would enter the analysis in the diffusion limited reaction term as indicated by earlier research into diffusion in disordered media [17]. In the next section we present a simple particle balance model and we show where such a roughness-related term would contribute. 


\section{The particle balance model}

\subsection{Model}

Particle balance at a target yields the following equation for hydrogen surface density, $N_{H}$ on the target surface:

$$
\frac{d N_{H}}{d t}=r_{A}-r_{H D}-r_{H_{2} E R}-r_{H_{2} L H}-r_{H T r p}+r_{H d T r p}
$$

The convention used is that $N$ stands for number densities and $r$ stands for the rate per unit area per sec and $E$ for the migration energy of a process. The subscripts indicate the species or source or sink mechanism. Subscript, $A$ stands for arrival, $H D$ for atomic desorption, $\mathrm{H}_{2} \mathrm{ER}$ is hydrogen molecule formation due to Eley-Rideal (ER) mechanism [18], $H_{2} L H$ stands for hydrogen molecule formation due to the Langmuir-Hishelwood (LH) mechanism [19], HTrp for hydrogen trapping at open bond sites and HdTrp for detrapping of trapped hydrogen atoms.

The Boltzmann factor for trapped particles yields the equation for hydrogen atomic desorption and detrapping.

$$
\begin{aligned}
& r_{H D}=N_{H} \omega_{o} e^{-E_{H D} / k_{B} T} \\
& r_{H d T r p}=N_{T r p} \omega_{o} e^{-E_{H d T r p} / k_{B} T}
\end{aligned}
$$


By comparing the area occupied by a hydrogen atom on the surface with the area of influence of an incoming hydrogen atom, the ER process is represented by

$$
r_{H_{2} E R}=r_{A} N_{H} \pi R_{o}^{2}
$$

By comparing the area covered by a hydrogen atom diffusing on the surface to the area occupied by a single hydrogen atom, we estimate the LH process by

$$
r_{H_{2} L H}=N_{H} N_{H} \pi 2 d D
$$

Here $\mathrm{D}$ is the surface diffusion coefficient given by $\omega_{o} L_{S D}^{2} \exp \left(-E_{S D} / k_{B} T\right)[8]$, where $L_{S D}$ is the average jump length of the hydrogen atom diffusing on a graphite surface with a surface migration energy $E_{S D} \cdot d$ is the dimensionality of the region where the diffusion is happening. The effect of surface roughness on diffusion limited reactions should be taken into account in the LH recombination mechanism. For the degree of roughness considered in our simulations the LH recombination term is larger and is the reason for the lower hydrogen density on the rough surface as seen in Fig.3. Further work is required to include this effect in the analytical model.

Using the number of surface jumps per second from the Boltzmann relation, and the probability of trapping per jump, the hydrogen atomic trapping rate is

$$
r_{H T r p}=N_{H} P_{\text {trap }} \omega_{o} e^{-E_{S D} / k_{B} T}
$$


This term is much smaller than the LH term since $P_{\text {trap }}$, the probability of trapping per surface jump, is 0.0075 [20].

\subsection{Results}

The following values for the various inputs like activation energies, jump distances after de-activation, minimum distance of approach before recombination were used in both the KMC simulation and the particle balance model: $E_{H D}=1.9 \mathrm{eV}, E_{S D}=0.9 \mathrm{eV}, E_{H d T r p}=$ $2.6 \mathrm{eV}, L_{S D}=34.4 \AA$ and $R_{o}=2 \AA$. We plot the results of the simple model for the approach to steady-state hydrogen density on the surface in Fig.4. Note that these results match the KMC results (Fig.2) pretty well except for the fact that the steady state for $900 \mathrm{~K}$ occurs before the steady state for $600 \mathrm{~K}$. This is because in our macroscopic particle balance model, a single desorption value of hydrogen is used. This single value is the statistical average over the time-step of the various desorption mechanisms from the surface (atomic desorption, and molecular desorption via ER and LH mechanisms). This is not the case in the particle picture of KMC where these desorption mechanisms are resolved separately and is the reason for the fluctuations in Fig.2.

We then test the particle balance model with the results for hydrogen recombination rates from a more complex KMC model for hydrogen recycling described in [6]. The full 3-D KMC model had bulk deposition, diffusion and trapping in the bulk, diffusion from the bulk into the surface and vice-versa. The comparison is shown in Fig.5. The particle balance model qualitatively matches the results from the full $\mathrm{KMC}$ model for high fluxes. 
The rise at low temperatures for the low incident flux case could only be mimicked by

- artificially suppressing the ER term in the particle balance model, and

- increasing the migration energy for diffusion in the LH term from $0.9 \mathrm{eV}$ used for surface diffusion to $2.6 \mathrm{eV}$ being typical for bulk diffusion.

The above modifications are necessary because the particle balance model does not have losses to the bulk from the incident flux. Therefore it has an artificially high (unrealistic) estimate of hydrogen density on the surface, which overestimates the ER and LH contributions (see Eqns.4 and 5). An extension of the particle balance model including bulk effects is planned to overcome these deficiencies.

\section{Summary and conclusions}

KMC results [6] show that reactive-diffusive transport in porous internal surfaces plays an important part in understanding hydrogen recycling and retention in porous graphites and co-deposits. We used KMC to focus on the diffusion limited reactions on rough surfaces. A simple particle balance has been developed to understand the role of different mechanisms involved.

The particle balance model helps to understand the importance of different mechanisms in the simulations:

- The effect of surface roughness must be reflected in the diffusion limited reaction term (LH mechanism) of the particle balance model. 
- Bulk processes must be included in the particle balance model to match the full KMC results.

The combination of the code and the analytical model allowed us to explain:

- why a steady state hydrogen saturation of surface occurs faster at $600 \mathrm{~K}$ than at $900 \mathrm{~K}$ : This is because desorption, which has an activation energy of $1.9 \mathrm{eV}$ and is a slow process, becomes more and more important at higher temperatures delaying the approach to a steady state.

- why the steady state hydrogen density on a rough surface is lesser than that for a smooth surface: The effect of surface roughness on diffusion limited reactions leads to a larger diffusion and the Langmuir-Hishelwood recombination term gets larger.

\section{Acknowledgment}

R. Schneider and A. Rai acknowledge funding of the work by the Initiative and Networking Fund of the Helmholtz Association. M. Warrier acknowledges funding from the MaxPlanck India Fellowship.

\section{References}

[1] W. Eckstein. Computer simulations of ion-solid interactions. Springer series in material science 10, Springer-Verlag, (1991).

[2] Mark T. Robinson. Phys. Rev. B, 40(16) (1989) 10717-10726.

[3] A. A. Haasz, P. Franzen, J. W. Davis, S. Chiu, and C. S. Pitcher. J. Appl. Phys., 77(1) 
(1995) 66-86.

[4] Wolfhard Möller. J. Nucl. Mater., 162-164 (1989) 138-150.

[5] G. Federici and C.H. Wu. J. Nucl. Mater., 186 (1992) 131-152.

[6] A. Rai, R. Schneider, and M. Warrier. J. Nucl. Mater., 374 (2008) 304-312.

[7] M. Warrier, R. Schneider, E. Salonen, and K. Nordlund. J. Plasma Phys., 72(06) (2006) 799-804.

[8] M. Warrier, R. Schneider, E. Salonen, and K. Nordlund. Nucl. Fusion, 47 (2007) 1656-1663.

[9] A. Rai. Dynamic monte carlo modeling of hydrogen isotope reactive diffusive transport in porous graphite. Ph.D Thesis, Ernst Moritz Arndt Universitat(Greifswald, Germany) (2008).

[10] R. A. Causey, M. I. Baskes, and K. L. Wilson. J. Vac. Sci. Technol. A, 4(3) (1986) 1189-1192.

[11] M. Warrier, R. Schneider, E. Salonen, and K. Nordlund. J. Nucl. Mater., 337-339 (2005) $580-584$.

[12] K. Ashida, K. Ichimura, M. Matsuyama, and K. Watanabe. J. Nucl. Mater., 128 \& 129 (1984) 792 .

[13] T. R. Waite. Phys. Rev., 107(2) (1957).

[14] M. V. Smoluchowski. Z. physik. Chem., 92 (1917) 192.

[15] K.A. Fichthorn and W.H. Weinberg. J, Chem. Phys., 95(2) (1991) 1090-1096.

[16] A. B. Bortz, M. H. Kalos, and J. L. Lebowitz. J. Comput. Phys., 17 (1975) 10.

[17] S. Havlin and D. Ben-Avraham. Adv. Phys., 51(1) (2002) 187-292. 
[18] D. D. Eley and E. K. Rideal, Nature (London), 146 (1940) 401-402.

[19] I. Langmuir Trans. Faraday Soc. 17 (1922) 607-620; C. N. Hinshelwood, Ann. Res. London Chem. Soc., 27 (1930) 11.

[20] Pascale Roubin. University of Marseilles. Measurement of active carbon sites in co-deposits, Personal communication, (2007). 


\section{List of Figures}

1 Schema of the model to study reactive diffusive transport on rough surfaces

2 Approach to steady state hydrogen densities on the surface from KMC

3 Comparison of hydrogen retention for smooth and rough surfaces at 900 K from KMC

4 Approach to steady state hydrogen densities on the surface using the particle balance model

5 Comparison of hydrogen recombination rates from (i) the simple particle balance model with that from (ii) KMC 


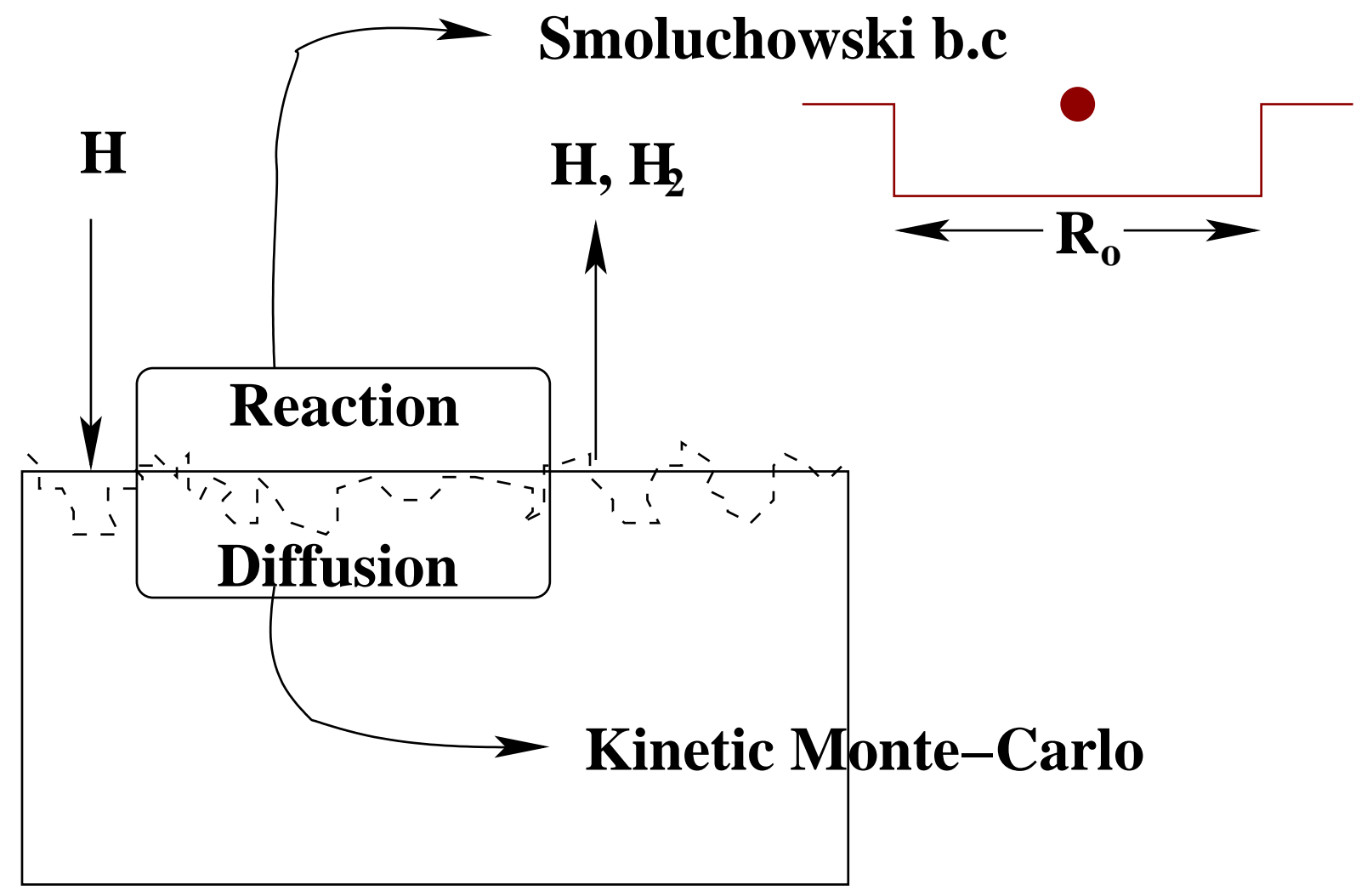

Fig. 1. Schema of the model to study reactive diffusive transport on rough surfaces 


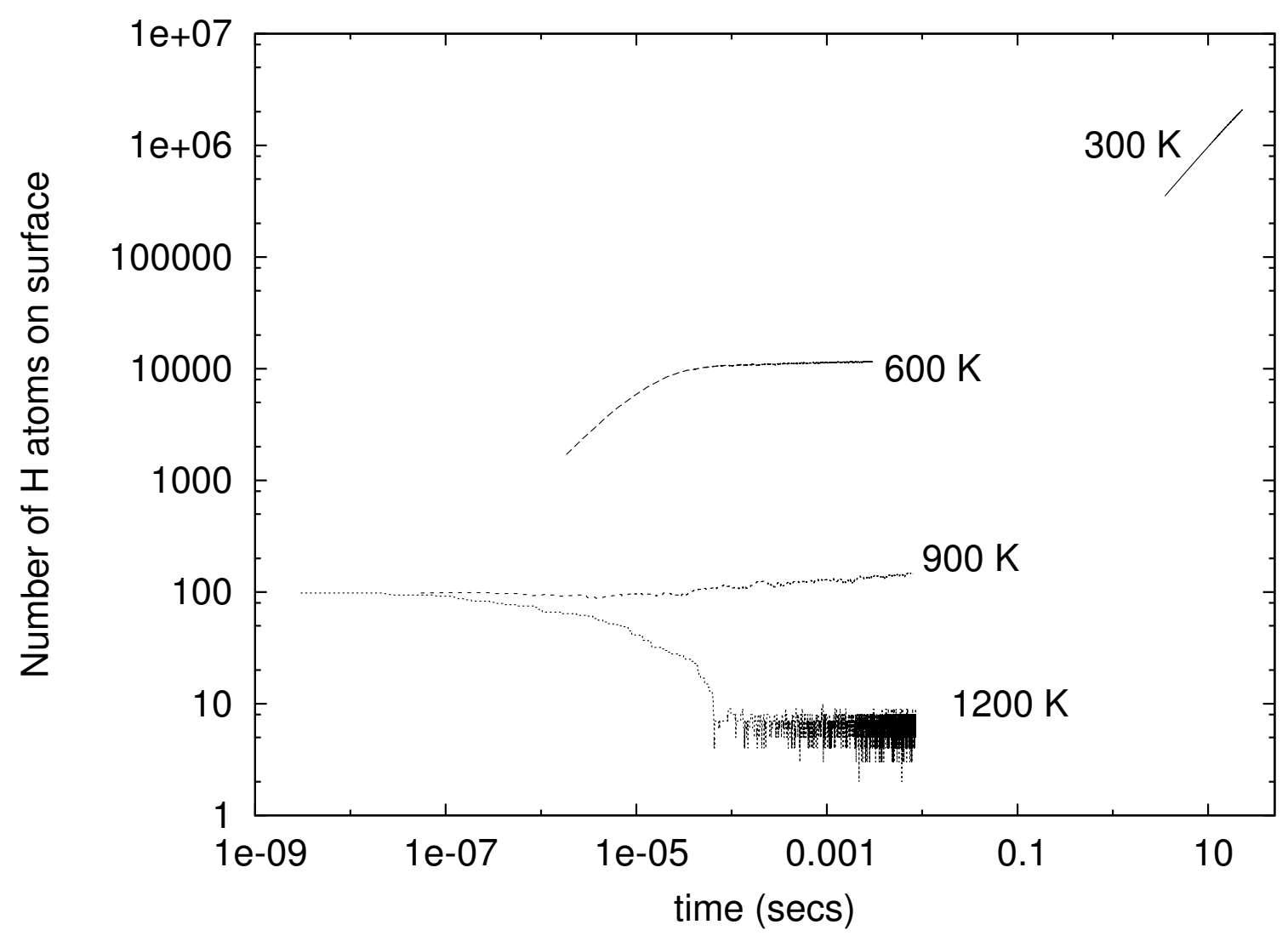

Fig. 2. Approach to steady state hydrogen densities on the surface from KMC 


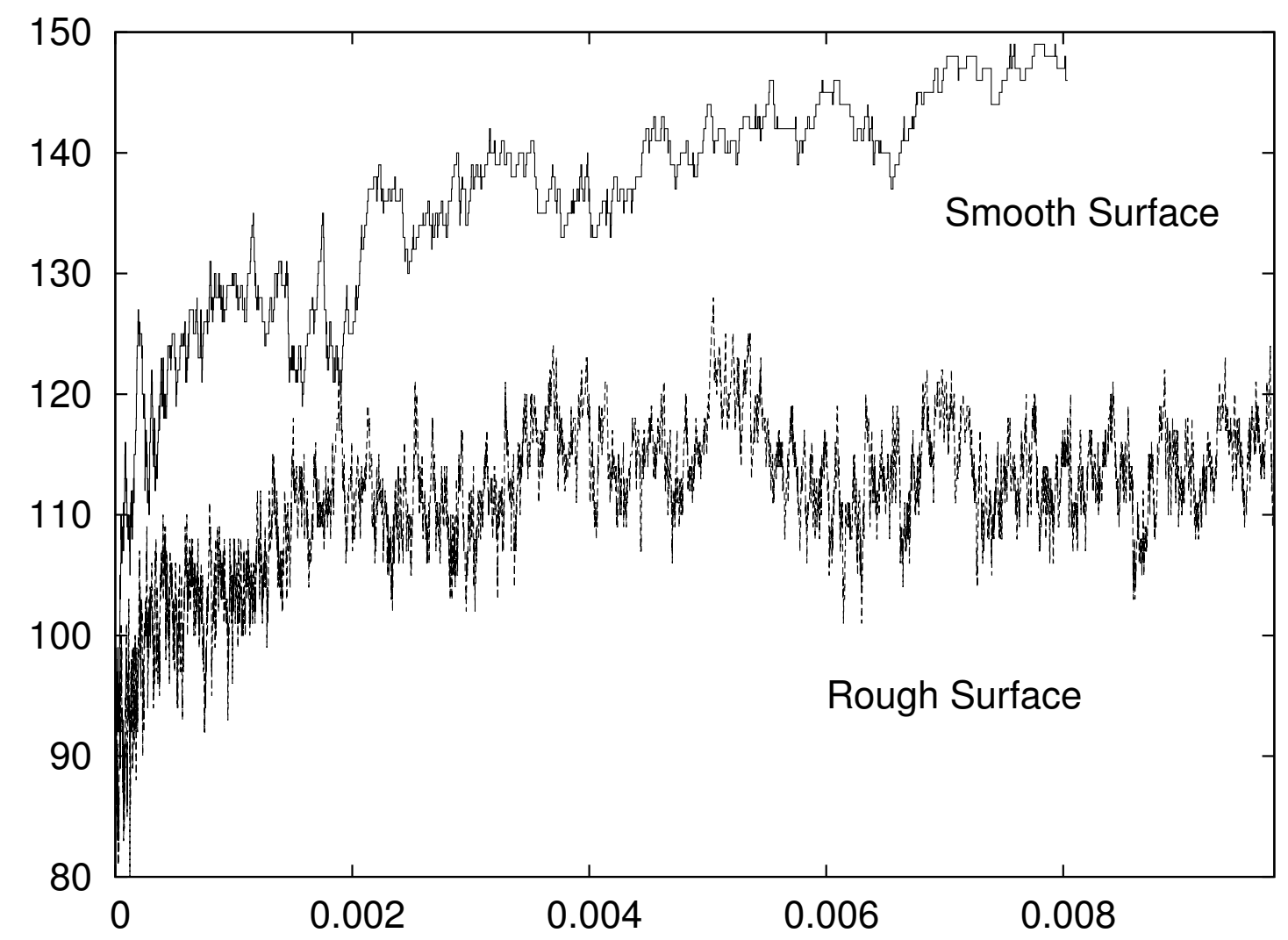

Fig. 3. Comparison of hydrogen retention for smooth and rough surfaces at $900 \mathrm{~K}$ from KMC 


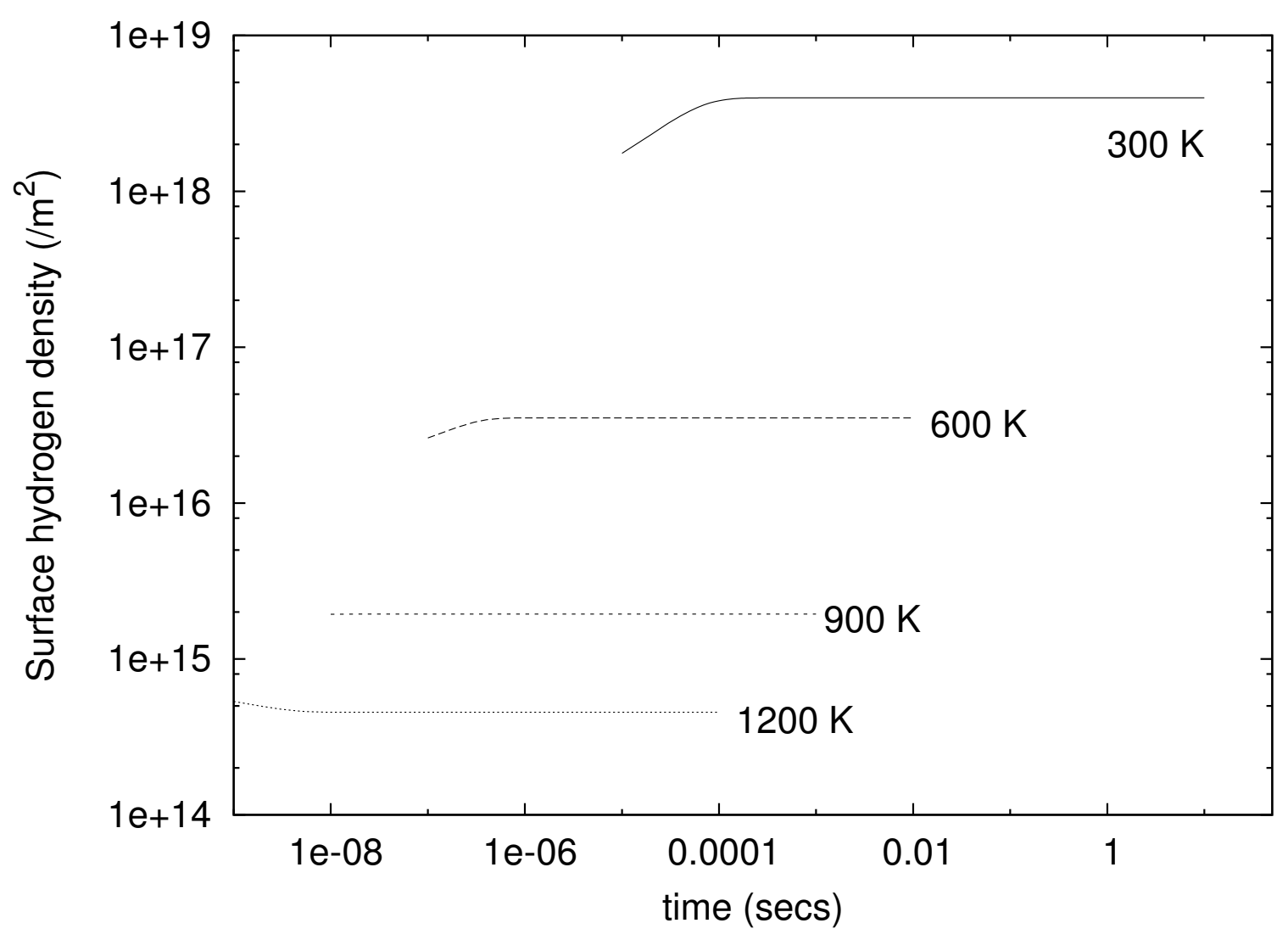

Fig. 4. Approach to steady state hydrogen densities on the surface using the particle balance model

(i) Simple particle balance model

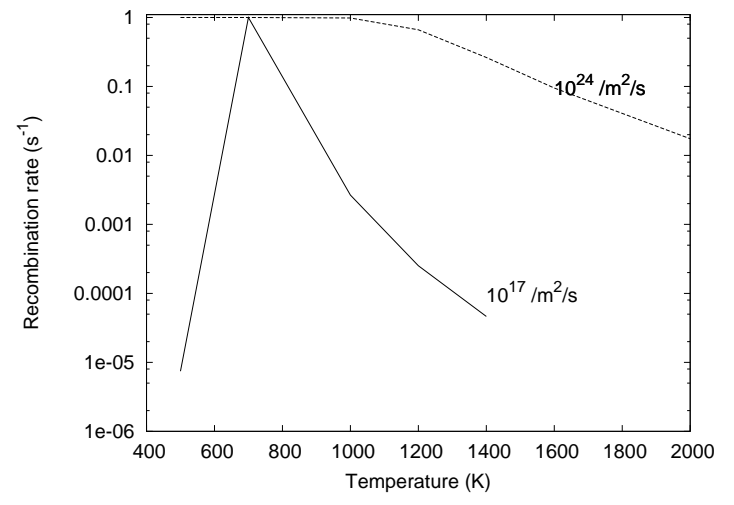

(ii) KMC model

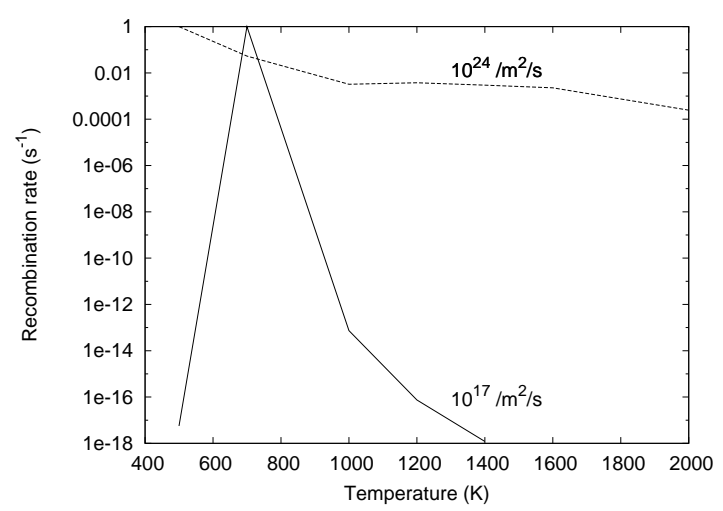

Fig. 5. Comparison of hydrogen recombination rates from (i) the simple particle balance model with that from (ii) KMC 